\title{
UNIVERSITY OF GRANADA RADIOCARBON DATES V
}

\section{CECILIO GONZALEZ-GOMEZ and PURIFICACION SANCHEZ-SANCHEZ}

Laboratorio de Datación por Carbono-14 and Instituto Andaluz de Geología Mediterránea (IAGM) del CSIC, Facultad de Ciencias, Universidad de Granada, E-18071 Granada, Spain

\section{INTRODUCTION}

This paper includes some determinations of archaeological, art and palaeobotanical samples from Spain and Portugal, obtained at the University of Granada Radiocarbon Dating Laboratory, mostly from 1986 to 1988. Pretreatment of charcoal and wood samples is a standard acid-basic procedure using $8 \% \mathrm{HCl}$ and $2 \% \mathrm{NaOH}$ at boiling temperature. The collagen of bone samples is obtained by the Longin (1971) method.

The method of dating is benzene synthesis and liquid scintillation counting as previously reported (González-Gómez, López-González \& Domingo-García 1982; González-Gómez, Sánchez-Sánchez and Domingo-García 1985; González-Gómez, Sánchez-Sánchez and Villafranca-Sánchez 1986, 1987). ${ }^{14} \mathrm{C}$ activity was measured in a Packard Tri-Carb Mod 4640 liquid scintillation spectrometer, using $20 \mathrm{ml}$ low ${ }^{40} \mathrm{~K}$ counting vials with $5 \mathrm{ml}$ benzene and $10 \mathrm{ml}$ PPO-toluene as scintillator with a background of $c a .9 \mathrm{cpm}$. Efficiency was approximately $70 \%$ using the part of spectrum above the end point of tritium.

Dates reported here are based on 0.95 of the activity of NBS oxalic acid modern standard and the Libby ${ }^{14} \mathrm{C}$ half-life of 5568 years. Samples were measured for 100 minutes repeating 30-40 times, as well as background and standard vials. The standard deviation quoted includes only $1 \sigma$ of the counting statistics of background, sample and modern standard counts. Calculations and data are processed by a PC computer, using extended programs made by González-Gómez (Computerextended programs for radiocarbon dating laboratories, ms. in preparation). Calibrated ages for a 2 o interval, are obtained by the method of Pearson et al. (1986), running the computer programs CALIB (Stuiver \& Reimer 1986) and CALI (González-Gómez, CALI, a computer program for calibration of radiocarbon dates, ms. in preparation) using the bidecadal curve for atmospheric origin samples. $\delta^{13} \mathrm{C}$ corrections are based on data reported by Stuiver and Polach (1977).

Sample descriptions are based on information supplied by those who submitted samples.

\section{ACKNOWLEDGMENTS}

The authors wish to express their thanks to the Instituto de Conservación y Restauración de Bienes Culturales, Departamento de Arqueología, Ministerio de Cultura, Madrid for their financial support to improve the Laboratory endowment.

\section{ARCHAEOLOGICAL SAMPLES}

Spain

\section{El Acequión series}

Samples from El Acequión ( $\left.39^{\circ} 01^{\prime} \mathrm{N}, 2^{\circ} 01^{\prime} \mathrm{W}\right)$, Albacete. Coll. $1985-1986$ and subm. 1987 by M. D. Fernández-Posse and C. Martín-Morales, Ministry of Cultura, Madrid, to date Bronze Age in E La Mancha. 
UGRA-265. AC-786-24a

$3770 \pm 80$

Charcoal from depth 1.86 m; cal. B.C. 2460-1970.

UGRA-266. AC-786-24b

$3680 \pm 80$

Charcoal from depth 1.86 m; cal. B.C. 2320-1880.

UGRA-271. AC-786-24c

$3730 \pm 100$

Charcoal from depth 1.86 m; cal. B.c. 2460-1890.

UGRA-302. AC-285-40

$5010 \pm 150$

Charcoal from depth 1.42 m; cal. B.C. 4224-3386.

UGRA-304. AC-286-71

$3790 \pm 120$

Charcoal from depth 2.52 m; cal. B.C. 2577-1890.

UGRA-307. AC-285-4

$3020 \pm 90$

Charcoal from depth $0.80 \mathrm{~m}$; cal. B.C. $1509-1000$.

UGRA-309. AC-785-8

$2990 \pm 90$

Charcoal from depth $0.80 \mathrm{~m}$; cal. B.C. $1440-942$.

General Comment: dates agree with archaeol. expectation, in study of last stage of occupation of site.

\section{Cabezo del Cuervo series}

Samples from Cabezo del Cuervo $\left(41^{\circ} 03^{\prime} \mathrm{N}, 0^{\circ} 08^{\prime} \mathrm{W}\right)$, Alcañiz, Teruel prov. Coll. 1982 by J. Vicente-Redón and subm. 1984 by P. Atrián-Jordán, Mus. Archaeol. Teruel, to date different cultural levels in middle valley of Ebro River.

UGRA-215. No. 23

$3410 \pm 90$

Wood from depth $1.20 \mathrm{~m}$; cal. B.C. $1950-1520$.

UGRA-216. No. 37

$3450 \pm 90$

Wood from depth $1.50 \mathrm{~m}$; cal. B.C. 2028-1530.

UGRA-228. No. 15

$3320 \pm 90$

Wood from depth $0.70 \mathrm{~m}$; cal. B.C. $1878-1420$.

UGRA-229. No. 53

$3420 \pm 90$

Wood from depth $2.37 \mathrm{~m}$; cal. B.C. $1960-1520$. 
UGRA-230. No. 47

Wood from depth $1.75 \mathrm{~m}$; cal. B.C. 1734-1310.

UGRA-239. No. 6

Wood from depth $0.63 \mathrm{~m}$; cal. B.C. $1880-1430$.

UGRA-240. No. 31

Wood from depth $1.35 \mathrm{~m}$; cal. B.C. 1960-1328.

UGRA-269. No. 44

$3230 \pm 80$

Wood from depth $1.72 \mathrm{~m}$; cal. B.C. 1731-1324.

General Comment: dates agree with archaeol. expectation.

\section{El Castillo de Burgos series}

Samples from El Castillo de Burgos (Burgos Castle) $\left(42^{\circ} 20^{\prime} \mathrm{N}, 3^{\circ} 43^{\prime} \mathrm{W}\right)$. Coll. 1985 by J. M. Martínez-González and subm. 1985 by J. L. de Uribarri-Angulo to date cultural stages at end of Bronze Age and first Iron Age.

\section{UGRA-226. No. 9 Nivel XII}

$2900 \pm 100$

Charcoal from depth $2.08 \mathrm{~m}$; cal. B.C. 1410-840.

UGRA-227. SII-NI-M1

$$
2710 \pm 80
$$

Seeds from depth $1.52 \mathrm{~m}$; cal. B.c. 1040-790.

General Comment: expected age was ca. $2720 \pm 250$ B.P.

\section{Cerrillo Blanco}

Charcoal from Cerrillo Blanco $\left(37^{\circ} 52^{\prime} \mathrm{N}, 4^{\circ} 11^{\prime} \mathrm{W}\right)$, Porcuna, Jaén prov. Interlaboratory check sample subm. 1988 by Barcelona Radiocarbon Dating Laboratory.

UGRA-280. MIP IAB-58/59

$2530 \pm 80$

Comment: UBAR date was $2650 \pm 90$; cal. B.C. $830-400$.

\section{Cerro de la Mora}

Sample from Cerro de la Mora $\left(37^{\circ} 14^{\prime} \mathrm{N}, 3^{\circ} 59^{\prime} \mathrm{W}\right)$, Moraleda de Zafayona, Granada prov. Charcoal from depth $4 \mathrm{~m}$ coll. and subm. by J. Carrasco-Rús to date end of Bronze Age in an Argaric-type fortification. Other samples from Cerro de la Mora were dated previously by González-Gómez, Sánchez-Sánchez and Domingo-García (1985: 611), González-Gómez, Sánchez-Sánchez and Villafranca-Sánchez (1986: 1201, 1987: 384).

UGRA-283. $\quad \mathrm{Cm} / 87 /$ Fondo

$3030 \pm 80$

Comment: date agrees with expected age; cal. B.C. 1495-1030. 


\section{Dolmen de Azután}

Bones from Dolmen de Azután (39 $\left.45^{\prime} \mathrm{N}, 5^{\circ} 10^{\prime} \mathrm{W}\right)$, Azután, Puente del Arzobispo, Toledo prov. Sample from 1.70 m depth coll. 1983 and subm. 1985 by P. Bueno-Ramírez, Dept. Prehist., Univ. Alcalá de Henares, Madrid prov., to date megalithic chronology in Meseta.

UGRA-288. AZUTAN $5060 \pm 90$

Comment: expected age was ca. $4800 \pm 150$ B.P.; cal. B.C. 4038-3690.

\section{Ermita de San Fructuoso}

Sample from Ermita de San Fructuoso $\left(43^{\circ} 14^{\prime} \mathrm{N}, 4^{\circ} 17^{\prime} \mathrm{W}\right)$, La Miña, Ruente, Cantabria. Charcoal from $0.70 \mathrm{~m}$ depth coll. 1985 by E. van den Eynde Ceruti and subm. 1985 by M. A. GarcíaGuinea, Mus. Reg. Prehist. \& Archaeol. Santander. This date is important for correct interpretation of site.

\section{UGRA-270. C1T 2}

$1090 \pm 80$

Comment: expected age was ca. $1010 \pm 350$ B.P.; cal. A.D. $770-1113$.

\section{Les Grioteres}

Sample from Les Grioteres $\left(41^{\circ} 58^{\prime} \mathrm{N}, 2^{\circ} 23^{\prime} \mathrm{E}\right)$, Vilanova de Sau, Barcelona prov. Charcoal from $1.70 \mathrm{~m}$ depth coll. 1985 and subm. 1986 by J. Castany-Llussa, Inst. Prehist. \& Archaeol. Univ. Barcelona, to date first human occupation of site in Neolithic period.

\section{UGRA-274. G-N 7}

$5280 \pm 90$

Comment: expected age was ca. $5210 \pm 250$ B.P.; cal. B.C. 4340-3825.

\section{Los Panizales}

Sample from Los Panizales $\left(41^{\circ} 02^{\prime} \mathrm{N}, 0^{\circ} 09^{\prime} \mathrm{W}\right)$, Alcañiz, Teruel prov. Charcoal from $0.25 \mathrm{~m}$ depth coll. and subm. 1987 by J. A. Benavente-Serrano, Taller Arqueol Alcañiz. Sample from inner part of probable tumulus close to surficial lithic bed. Relation between both is doubtful. End of Neolithic period.

UGRA-299. P-1

$2310 \pm 90$

Comment: cal. B.C. $763-180$.

Portugal

Alto do Castelo

Wood from Aito do Castelo $\left(39^{\circ} 15^{\prime} \mathrm{N}, 8^{\circ} 35^{\prime} \mathrm{W}\right)$, Alpiarça, Santarém prov. Sample coll. 1982 by P. Kalb and subm. 1987 as interlaboratory comparison sample by the Lisbon Radiocarbon Dating Laboratory.

UGRA-281. AC-466 $2020 \pm 80$

Comment: ICEN-122: $2120 \pm 70$ B.P.; cal. B.C. 340 - cal. A.D. 130. 


\section{Barrocal. Alto series}

Charcoal from Barrocal Alto $\left(41^{\circ} 16^{\prime} \mathrm{N}, 6^{\circ} 33^{\prime} \mathrm{W}\right)$, Peredo da Bemposta, Mogadouro, Tras Os Montes prov. Coll. 1984 by M. de Jesus Sanches, A. Barroso, V. Lopes and A. Gomes-Sebre and subm. 1984 by M. de Jesus Sanches, Porto Archaeol. Study Group, to date prehistoric village.

UGRA-223. 1/BA-84/S-II/T.26-L

$3230 \pm 100$

Comment: expected age was ca. $3200 \pm 250$ B.P.; cal. B.C. $1740-1310$.

UGRA-272. 2/BA-84/S-II/U.26

$2050 \pm 100$

Cal. B.C. 370 - cal. AD 130.

Comment: age is younger than expected $-3200 \pm 250$ B.P.

UGRA-273. 3/BA-84/S-II/X.22

$6950 \pm 90$

Sample from depth $0.65 \mathrm{~m}$; cal. B.C. $6034-5630$.

Comment: age is older than expected $-3450 \pm 300$ B.P.

\section{Castro do Coto da Pena series}

Seeds from Castro do Coto da Pena $\left(41^{\circ} 52^{\prime} \mathrm{N}, 8^{\circ} 50^{\prime} \mathrm{W}\right)$, Vilarelho, Caminha, Viana do Castelo prov. Samples coll. 1983 and subm. 1984 by A. Coelho Ferreira da Silva, Univ. do Porto, to date last occupation of site at end of Bronze Age.

UGRA-200. No. 5

$2950 \pm 100$

Cal. B.C. $1430-900$.

UGRA-220. No. 2

$2920 \pm 110$

Sample from depth $1.80 \mathrm{~m}$; cal. B.C. $1420-840$.

General Comment: dates agree with archaeol. expectation that confirm age of occupation of bottom stratum of site.

\section{Mamoa do Monte da Olheira}

Charcoal from Mamoa do Monte da Olheira $\left(41^{\circ} 12^{\prime} \mathrm{N}, 8^{\circ} 02^{\prime} \mathrm{W}\right)$, Serra da Aboboreira, Baiâo, Dist. do Porto, Douro Litoral prov. Sample from depth 0.53 m, coll. 1981 and subm. 1987 by D. de Jesus da Cruz, to date megalithic necropolis with 40 tombs in region.

UGRA-287. Amostra no. 1

$5630 \pm 90$

Comment: expected age was ca. $4960 \pm 500$ B.P.; cal. B.C. $4720-4340$.

\section{Povoado Pre-Historico da Lavra}

Samples from Povoado Pre-Historico da Lavra $\left(41^{\circ} 10^{\prime} \mathrm{N}, 8^{\circ} 04^{\prime} \mathrm{W}\right)$, Lavra, Marco de Cavaneses, Soalhaês prov. Coll. 1982 and subm. 1984 by M. de Jesus Sanches to date village. 
UGRA-267. 1-LA.82/E.C.3

Charcoal to date village; cal. B.C. 4936-4490.

UGRA-268. 2-LA.82/F.4

$7500 \pm 100$

Charcoal to date silo or cave; cal. B.C. 6558-6110.

General Comment: expected ages were $3700 \pm 250$ B.P. for both samples.

\section{ART SAMPLES}

Spain

UGRA-67.

Wood from statue of St. Anthony the Abbot; expected age was ca. 15 th century A.D. Sample subm. by P. Gómez-Acha, Granada.

UGRA-141.

$290 \pm 90$

Wood from rear side of picture on plank; expected age was ca. 17th century A.D. Sample subm. 1983 by J. J. Pons-Marco, Castellón de la Plana.

UGRA-219.

$410 \pm 90$

Wood from rear side of St. Andrew picture on plank; expected age was ca. 14th century A.D. Sample subm. 1985 by J. L. Vílchez-Quero and J. Torres-Garzón, Granada.

\section{Palaeobotanical SaMples}

Spain

\section{Barrio de San Miguel series}

Samples from Cerro de San Miguel ( $\left.43^{\circ} 21^{\prime} \mathrm{N}, 1^{\circ} 48^{\prime} \mathrm{W}\right)$, Anzarán, Irún, Guipúzcoa prov. Coll. and subm. 1987 by J. M. Edeso-Fito and F. M. Ugarte-Elorza, Soc. Ciencias Aranzadi, Mus. S Telmo, San Sebastián, to date Quaternary littoral paleogeographic evolution on coast of Gulf of Vizcaya (Quipuzcoan sector).

UGRA-294. A3. $0.85 \mathrm{~m}$. (C1)

$>45,000$

Charcoal from depth $4.05 \mathrm{~m}$.

UGRA-301. Ao. $0.30 \mathrm{~m} .(\mathrm{C} 4)$

$38,300 \pm 2100$

Charcoal from depth $3.20 \mathrm{~m}$.

\section{Maxalen Berri}

Sample from Maxalen Berri $\left(43^{\circ} 08^{\prime} \mathrm{N}, 1^{\circ} 51^{\prime} \mathrm{W}\right)$, Oyarzum, Guipuzcoa prov. Coll. and subm. 1987 by J. M. Edeso-Fito and F. M. Ugarte-Elorza, Soc. Ciencias Aranzadi, Mus. S Telmo, San 
Sebastián, to date Quaternary evolution in Oyarzum River valley.

UGRA-300. OYA-1

$2550 \pm 90$

Wood from depth $2.00 \mathrm{~m}$; cal. B.c. $900-400$.

\section{Sopelana}

Sample from Sopelana $\left(43^{\circ} 23^{\prime} \mathrm{N}, 3^{\circ} 00^{\prime} \mathrm{W}\right)$, Sopela, Vizcaya prov. Coll. and subm. 1987 by F. M. Ugarte-Elorza to date Würm and Holocene evolution of paleolandscape on Vizcaya coast.

\section{UGRA-293. Sop-1}

$41,400 \pm 2500$

Wood from depth $3.00 \mathrm{~m}$.

\section{REFERENCES}

González-Gómez, C., López-González, J. D. and Domingo-García, M. 1982 University of Granada radiocarbon dates I. Radiocarbon 24(2): 217-221.

González-Gómez,C., Sánchez-Sánchez,P. and DomingoGarcía, M. 1985 University of Granada radiocarbon dates II. Radiocarbon 27(3): 610-615.

González-Gómez, C., Sánchez-Sánchez, P. and Villafranca-Sánchez, E. 1986 University of Granada radiocarbon dates III. Radiocarbon 28(3): 1200-1205.

1987 University of Granada radiocarbon dates IV. Radiocarbon 29(3): 381-388.

Longin, R. 1971 New method of collagen extraction for radiocarbon dating. Nature 230: 241-242.

Pearson, G. W., Pilcher, J. R., Baillie, M. G. L., Corbet, D. M. and Qua, F. 1986 High-precision ${ }^{14} \mathrm{C}$ measurements of Irish oaks to show the natural ${ }^{14} \mathrm{C}$ variations from $\mathrm{AD}$ 1840 to 5210 BC. In Stuiver, M. and Kra, R. S., eds., Proceedings of the 12 th International ${ }^{14} \mathrm{C}$ conference. Radiocarbon 28(2B): 911-934.

Stuiver, M. and Polach, H. 1977 Reporting of ${ }^{14} \mathrm{C}$ data. Radiocarbon 19(3): 355-363.

Stuiver, M. and Reimer, P. J. 1986 A computer program for radiocarbon age calibration. In Stuiver, M. and Kra, R. S., eds., Proceedings of the 12 th International ${ }^{14} \mathrm{C}$ conference. Radiocarbon 28(2B): 1022-1030. 
